This is the accepted version (i.e. peer-reviewed) of the following article: Andres, M., Masson, N., Larigaldie, N., Bonato, M., Vandermeeren, Y., \& Dormal, V. (2020). Transcranial electric stimulation optimizes the balance of visual attention across space. Clinical Neurophysiology, 131(4), 912-920. http://doi.org/10.1016/j.clinph.2019.12.415. This manuscript version does not replicate the final authoritative version of the article. Figures 1 to 5 appear at the end of the manuscript.
\end{abstract}

(C) 2020. This manuscript version is made available under the CC-BY-NC-ND 4.0 license http://creativecommons.org/licenses/by-nc-nd/4.0/ 


\title{
Transcranial electric stimulation optimizes the balance of visual attention across space
}

\author{
Michael Andres ${ }^{1,2}$, Nicolas Masson ${ }^{1,3}$, Nathanael Larigaldie ${ }^{1}$, Mario Bonato ${ }^{4,5}$, \\ Yves Vandermeeren ${ }^{2,6}$ and Valérie Dormal ${ }^{1}$
}
${ }^{1}$ Psychological Sciences Research Institute, Université catholique de Louvain, Belgium
${ }^{2}$ Institute of Neuroscience, Université catholique de Louvain, Belgium
${ }^{3}$ Department of Psychology, Faculty of Science, University of Tuebingen, Germany
${ }^{4}$ Department of Experimental Psychology, Ghent University, Belgium,
${ }^{5}$ Department of General Psychology, University of Padova, Italy
${ }^{6}$ Stroke Unit \& Neuromodulation Unit, Neurology Department, CHU UCL Namur - Godinne, Université catholique de Louvain, Belgium

Running title : Neuromodulation of Spatial Attention

\section{Corresponding author :}

Michael Andres

Psychological Sciences Research Institute

Université catholique de Louvain

Place Cardinal Mercier, 10

1348 Louvain-la-Neuve

Belgium

Email : michael.andres@uclouvain.be 


\section{Highlights}

- The study tested the potential benefit of bilateral tDCS for attention modulation

- Left anodal and right cathodal tDCS increased visual sensitivity in both fields

- TDCS may boost attention resources and facilitate functional rehabilitation 


\section{Abstract}

Objective. Transcranial direct current stimulation (tDCS) provides a way to modulate spatial attention by enhancing the ratio of neural activity between the left and right hemispheres, with a potential benefit for the rehabilitation of visual neglect.

Methods. We tested the effect of bilateral tDCS in healthy individuals performing a visual detection task. This protocol consists in the positioning of the anode and cathode on mirror positions over the left and right parietal areas. The stimulation was repeated over three days to maximize the chance to observe a bias to the hemispace controlateral to the anode.

Results. Compared to a sham treatment, left anodal - right cathodal stimulation enhanced attention across the full range of space, since the first day with no build-up effect on the next days, and modified the balance of left-right omissions when stimuli appeared at the same time. Conclusion. Bilateral tDCS improved detection in both visual fields, with no privileged processing of one side, except when concurrent stimuli were presented. The results provide partial support to the hemispheric rivalry hypothesis.

Significance. The technique has the potential to boost attention in neglect patients but should be used as an adjuvant rather than as an alternative to functional rehabilitation.

Keywords : stroke, hemineglect, parietal, attention, awareness, tDCS 


\section{Introduction}

Unilateral spatial neglect is a dramatic impairment of attention orientation which cannot be ascribed to an elementary sensorimotor deficit. The neuropsychological examination demonstrates privileged processing of the space ipsilateral to the damaged hemisphere, to the detriment of the contralateral space (Heilman et al., 1993; Rode et al., 2017). Extinction is observed when the patient has to pay attention to bilateral stimuli and only report the one on the ipsilesional side. Neglect is associated with poor hemiplegia recovery and functional autonomy limitation (Denes et al., 1982; Di Monaco et al., 2011; Farnè et al., 2004; Vossel et al., 2013) and continues to affect visuospatial behaviour, years after stroke, especially in divided attention conditions (Andres et al., 2019; Bonato et al., 2010; Bonato, 2015). There is thus a need for new rehabilitation methods that lead to efficient and durable recovery of symmetric spatial processing after stroke.

The development of non-invasive brain stimulation methods has opened up perspectives for the neurorehabilitation of patients with spatial disorders (Koch et al., 2013). In healthy individuals, transcranial magnetic stimulation (TMS) over the right posterior parietal cortex (PPC) modifies the activation state of the parietofrontal connectivity in the left hemisphere (Koch et al., 2011; Szczepanski and Kastner, 2013), through callosal connections (Chechlacz et al., 2015), and produces neglect-like or extinction-like phenomena in visual detection tasks (Dambeck et al., 2006; Hilgetag et al., 2001; Meister et al., 2006). These findings have corroborated the hypothesis that full space attention is achieved through reciprocal inhibition between the two hemispheres whose role is to orient attention in opposite directions (Kinsbourne, 1977). Further TMS studies showed that reducing the excitability of the left hemisphere of right-brain-lesioned patients attenuates left neglect (Koch et al., 2008; 2012; Oliveri et al., 2001; Song et al., 2009). Increasing the excitability of the lesioned hemisphere 
with high-frequency repetitive TMS is a complementary way to achieve this goal but with more incomfort and a higher risk of inducing a seizure. These issues can be circumvented by using transcranial direct current stimulation (tDCS) that modifies the state of neuronal excitability without causing depolarization. The principle of using tDCS to rehabilitate visuospatial attention after unilateral brain lesion is supported by some promising results (Brem et al., 2014; Ko et al., 2008; Sparing et al., 2009) but the mechanism underlying the effect of tDCS on visual attention remains unclear, limiting the development of efficient neurorehabilitation strategies (Fan et al., 2018; Salazar et al., 2018). In patients, directional biases were generally measured with paper-and-pencil tests, such as line bisection, providing little insight into attention orienting mechanisms. In healthy humans, a seminal study showed that anodal or cathodal parietal stimulation respectively facilitates or deteriorates detection of controlateral targets, irrespective to the stimulated hemisphere (Sparing et al., 2009). But other studies concluded to a general enhancement of visual attention, for example after right cathodal stimulation, without any advantage for either hemifield (Moos et al., 2012; Weiss and Lavidor, 2012). These discrepant results question the relevance of the hemispheric rivalry hypothesis for making predictions about the outcome of neurorehabilitation strategies using tDCS in stroke patients. A source of discrepancy may lie in the use of unilateral stimulation to address the issue of hemispheric rivalry. Unilateral stimulation implies that one electrode is placed over the PPC while the other electrode is placed over a remote site that could differ between studies. The remote electrode is wrongly considered as a non-relevant, while the neuromodulation of the brain areas covered by this other electrode might actually intervene in the inter-hemispheric crosstalk.

The present study investigates the effect of bilateral tDCS on visuospatial attention. Bilateral tDCS offers an alternative and potentially more efficient way to modify the interhemispheric balance and clarify its relationship with attention orientation. The placement of the 
electrodes on mirror positions on the left and right PPC allows combining the excitatory effect of anodal stimulation over one hemisphere with the inhibitory effect of cathodal stimulation over the opposite hemisphere. So far, three out of four studies have reported a positive outcome on neglect measures when the cathode was placed over the left hemisphere and the anode over the right hemisphere of right-brain-lesioned patients. Two randomized controlled trials combined bilateral tDCS with visual training and showed improved performance compared to a control group receiving conventional therapy (Bang and Bong, 2015; Turgut et al., 2018). One cross-over double-blind study found no effect of bilateral tDCS in any of the conventional subtests of the Behavioural Inattention Test (Smit et al., 2015), while another showed a significant reduction of righward bias in line bisection compared to sham and unilateral parietal tDCS (Sunwoo et al., 2013). Note that in the latter study, bilateral tDCS was achieved with two circuits so that a cathode was also placed over the left supraorbital area and an anode over the right supraorbital area (Sunwoo et al., 2013). Finally, aside from one single case study that monitored visuospatial performance over several days (Brem et al., 2014), not much is known about the stability of tDCS after-effects and their persistence after the last session. The same holds for studies in healthy participants where the implementation of multiple sessions served to counterbalance the order of the tDCS conditions (Moos et al., 2012; Sparing et al., 2009). We aimed to complement this research by investigating the effects of bilateral tDCS, with two opposite electrode montages, in healthy participants performing a computerized detection task. The present study extended over five consecutive days, including a pre-treatment session, three tDCS sessions replicating the same protocol to test the stability of the effects, and a posttreatment session taking place $24 \mathrm{~h}$ after the last tDCS session. Healthy participants were asked to detect left, right or bilateral targets whose size was calibrated to their individual detection threshold. Detection was performed while an electrical direct current was applied between the left and the right PPC with two opposite montages, i.e., left anode - right cathode ( $\mathrm{L}_{\mathrm{ANODE}}-$ 
$\mathrm{R}_{\text {CAthode }}$ group) and left cathode - right anode (LCATHODE-R $\mathrm{R}_{\text {ANOde }}$ group), or faded after 30 seconds resulting in a sham stimulation (sham group). We first examined visual sensitivity, indexed by the number of hits and false alarms, and then investigated neglect-like asymmetries by computing the detection rate in left and right unilateral trials. According to the hemispheric rivalry hypothesis, participants in the $\mathrm{L}_{\mathrm{ANODE}}-\mathrm{R}_{\mathrm{CATHODE}}$ group should prioritize right target processing whereas participants in the $\mathrm{L}_{\text {CATHODE }}-\mathrm{R}_{\mathrm{ANODE}}$ group should prioritize left target processing (Figure 1A). An additional analysis was performed on bilateral trials to measure extinction-like effects, i.e., a relative increase in the number of left vs. right omissions when the two targets were presented simultaneously.

\section{Methods}

\subsection{Participants}

Thirty-six healthy volunteers (18 females, age range: 20 to 30 years) provided written informed consent to take part in the experiment. All participants declared themselves as righthanded, were naive to the hypothesis being tested, had normal or corrected-to-normal vision and reported no history of neurological disorder, and had no intake of drugs modulating brain activity. We replaced one participant because he never reported the right target in bilateral trials, and another one because an encoding error caused the loss of his data for one tDCS session. The experimental protocol was approved by the ethics committee of the Université catholique de Louvain ( ${ }^{\circ}$ B40320108544, Comité d'Ethique Hospitalo-Facultaire Saint-Luc-UCL).

\subsection{Transcranial direct current stimulation}


Bilateral tDCS was delivered over the parietal areas through a battery-driven electrical stimulator (DC Stimulator Plus, NeuroConn). Two electrodes $(5 \times 7 \mathrm{~cm}$, encased in salinesoaked synthetic sponges) were placed over the left (P3) and right (P4) PPC, with the main axis oriented along the rostro-caudal axis. Participants were blinded to group allocation. The $\mathrm{L}_{\text {ANODE}}-\mathrm{R}_{\mathrm{CATHODE}}$ group $(\mathrm{N}=12)$ performed the task with the anodal electrode placed over P3 and the cathodal electrode over P4 to prioritize right target detection, whereas the L $\mathrm{CATHODE}^{-}$ $\mathrm{R}_{\text {ANODE }}(\mathrm{N}=12)$ performed the task with the reverse electrode montage. The control group $(\mathrm{N}=12)$ performed the visual detection task in the same conditions while receiving sham tDCS. For the real stimulation, a constant current of $1.5 \mathrm{~mA}$ was delivered for 20 minutes. Sham stimulation was programmed with the same parameters except that the stimulator was turned off progressively after 30 seconds. Baseline performance was measured 24 hours pre-treatment; then the bilateral-tDCS protocol was repeated over three consecutive days; and the posttreatment effect was measured 24 hours after the last tDCS session.

\subsection{Task and Stimuli}

While they were receiving tDCS, participants had to detect black targets flashed over a grey background in the left and/or right visual field using the Psychopy software (Peirce, 2007). They sat at a distance of $60 \mathrm{~cm}$ from the computer screen. Their head rested on a chin rest and their hands lied on the table with the right index, middle and ring fingers placed respectively on the left, down and right keys of a keyboard. Targets were flashed on the screen with an eccentricity of $20^{\circ}$ and two possible peri-threshold sizes selected after calibration during the pre-treatment session. The calibration block counted 20 trials for each combination of trial type (left, right or bilateral) and pixel size $(2 \times 2,2 \times 3,3 \times 3,3 \times 4,4 \times 5)$, which were randomly intermixed with 20 catch trials (no target). The two peri-threshold sizes corresponded to 
adjacent pixel sizes (e.g., $3 \times 3$ and $3 \times 4$ ) giving a detection rate respectively lower and higher than $50 \%$, thus ensuring that the task engaged a high level of attention resources (Figure 1B). They were defined separately for left and right targets. The performance in unilateral trials served to calibrate the size of left and right targets in bilateral trials as well. This procedure was adopted from previous TMS (Dambeck et al., 2006; Hilgetag et al., 2001; Meister et al., 2006) and tDCS (Sparing et al., 2009) studies. After calibration, the average size of left targets $(M=$ 9.5, $S D=2.5$ pixels $)$ was slightly smaller than the average size of right targets $(M=10.6, S D=$ 3.3 pixels; $\left.F(1,33)=5.8, p=.022, \eta_{\mathrm{p}}^{2}=.15\right)$, but there was no difference between groups $\left(F(1,33)=1.25, p=.3, \eta_{\mathrm{p}}^{2}=.07\right)$. The target followed the fixation cross after $500 \mathrm{msec}$ and remained on the screen for $50 \mathrm{msec}$. The next trial started $1000 \mathrm{msec}$ after the $2000-\mathrm{msec}$ deadline allowed for responding. During each tDCS session (day 1 to 3), participants performed 320 trials of the visual detection task, with 40 trials for each combination of trial type (left, right or bilateral) and threshold (infra- $v s$. supra-) and 80 additional catch trials. The total duration of the task corresponded to the total duration of the tDCS (20 min). During the pre- and posttreatment sessions, baseline performance and after-effects were estimated by means of 160 trials of the same visual detection task.

\subsection{Data analysis}

The analyses were conducted after excluding trials with a response time falling outside a $200-1100$ ms range defined arbitrarily after visual inspection of the RT distribution $(0.6 \%$ of trials). In the remaining trials, we first looked at the neuromodulation effect on the visual sensitivity index, which takes into account both the correct detection rate and the false alarm rate. The sensitivity index (d') was computed using the following formula : $z$ (hits) $-z$ (false alarms). In conditions where the hit or false alarm rate was equal to 0 or 1 , it was estimated 
through $1 /(2 * \mathrm{~N})$ or $1-1 /(2 * \mathrm{~N})$ before applying the inverse of the cumulative distribution function of the Gaussian distribution. The d' were entered in an ANOVA with TDCS ( $\mathrm{L}_{\mathrm{ANODE}}{ }^{-}$ $\mathrm{R}_{\mathrm{CATHODE}}, \mathrm{L}_{\mathrm{CATHODE}}-\mathrm{R}_{\mathrm{ANODE}}$, or sham) as a between-subject variable and TIME (pre-treatment, day 1 , day 2 , day 3 , or post-treatment) as a within-subject variable. This first analysis made no distinction between left and right for reasons of statistical independence. It was not possible to factor our the effect of hemifield in the visual sensitivity analysis because left and right false alarms should be treated as independent distributions whereas they are computed over the same sample of catch trials. Along the same lines, partial omissions in bilateral trials, i.e., "only left" or "only right" responses, should be treated as correct hits for one side of space and as misses for the other side of space whereas they refer to the same trials. To investigate neglect-like effects, we conducted a second ANOVA on the percentage of correct responses with TDCS ( $\mathrm{L}_{\mathrm{ANODE}}-\mathrm{R}_{\mathrm{CATHODE}}, \mathrm{L}_{\mathrm{CATHODE}}-\mathrm{R}_{\mathrm{ANODE}}$, or sham) as a between-subject variable, and TRIAL TYPE (left unilateral, right unilateral, bilateral, or catch trial) and TIME (pre-treatment, day 1, day 2, day 3 , or post-treatment) as within-subject variables. In a third step, we looked at the response bias in bilateral trials, commonly used to assess visual extinction, by computing the percentage of left (vs. right) responses among the partial omissions of the participants. The bias was expressed as a function of a difference score relative to the baseline performance and analyzed through an ANOVA with TDCS (L $\mathrm{L}_{\mathrm{ANODE}}-\mathrm{R}_{\mathrm{CATHODE}}, \mathrm{L}_{\mathrm{CATHODE}}-\mathrm{R}_{\mathrm{ANODE}}$, or sham) and DAY (day 1, day 2, or day 3) as factors. Post-hoc comparisons between groups were corrected using the Holm-Bonferroni procedure (JASP Team, 2016; jasp-stats.org). The partial $\eta^{2}$ and Cohen's $d$ were used as effect size estimates. The frequentist approach was complemented by Bayesian hypothesis testing using the Bayes Factor (BF) obtained by dividing the likelihood of the alternative hypothesis by that of the null hypothesis (Wagenmakers et al., 2018). BF varied between 0 and $\infty$ where values below 1 provide increasing evidence in favour of the null hypothesis and values above 1 increasing evidence for the alternative hypothesis. 


\section{Results}

\subsection{Visual sensitivity}

The analysis of the d' values over sessions revealed a significant effect of TIME $\left(F(2.85,103.77)=7.4, p<.001, \eta_{\mathrm{p}}^{2}=.18\right)$, which was best described by a quadratic trend $\left(F(1,33)=26.71, p<.001, \eta_{\mathrm{p}}^{2}=.45\right)$, demonstrating a sharp increase from pre-treatment to day 1, a plateau on day 2 and day 3, and a slight decrease post-treatment (Figure 2). The increase in visual sensitivity was observed in each group but it was more pronounced in the $\mathrm{L}_{\mathrm{ANODE}}-$ $\mathrm{R}_{\text {CATHODE }}$ group. There was indeed a main effect of $\operatorname{TDCS}\left(F(2,33)=4.12, p=.025, \eta_{\mathrm{p}}{ }^{2}=.2\right)$, and an interaction between TIME and TDCS $\left(F(6.29,103.77)=2.15, p=.051, \eta_{\mathrm{p}}^{2}=.12\right)$. Posthoc comparisons indicated that, while the three groups did not differ pre-treatment (all $p$-values $>$.1), the $\mathrm{L}_{\mathrm{ANODE}}-\mathrm{R}_{\mathrm{CATHODE}}$ group performed significantly better than the sham group on day 1 $(t(22)=2.69, p=.033, d=1.12)$, on day $2(t(22)=2.52, p=.034, d=0.42)$ and marginally on day $3(t(22)=2.37, p=.071, d=0.98)$. No difference was found between the $\mathrm{L}_{\mathrm{CATHODE}}-\mathrm{R}_{\mathrm{ANODE}}$ group and the sham group over the three days of stimulation (all $p$-values $>.1$ ). On the day after treatment, performance returned close to baseline so that none of the groups having received real tDCS differed from the sham group (all $p$-values $>.1$ ).

In line with these results, the Bayesian analyses revealed that the best model included the main effect of TIME, the main effect of TDCS and the TIME by TDCS interaction (BF $=1934)$. Moreover, the comparison of models that contain the TIME by TDCS interaction with models that only contain the main effects yielded a $\mathrm{BF}=3.77$, which constitutes moderate evidence for the interaction hinting at possible group differences during the tDCS sessions. The direct comparison of the $\mathrm{L}_{\mathrm{ANODE}}-\mathrm{R}_{\mathrm{CATHODE}}$ group and the sham group yielded BFs $(\mathrm{H} 1 / \mathrm{H} 0)$ of 0.42 pre-tDCS, 4.55 on day $1,4.48$ on day $2,2.6$ on day 3 , and 0.66 post-tDCS. The comparison of 
the $\mathrm{L}_{\mathrm{CATHODE}}-\mathrm{R}_{\mathrm{ANODE}}$ group and sham group yielded BFs (H1/H0) of 0.69 pre-tDCS, 0.37 on day $1,0.39$ on day $2,0.45$ on day $3,0.72$ post-tDCS. According to Jeffreys (1961), these values indicate moderate evidence for the hypothesis that $\mathrm{L}_{\mathrm{ANODE}}-\mathrm{R}_{\mathrm{CATHODE}}$ stimulation increased visual sensitivity, and anecdotal evidence for a null effect of $\mathrm{L}_{\mathrm{CATHODE}}-\mathrm{R}_{\mathrm{ANODE}}$ stimulation.

\subsection{Detection rate}

The analysis of the detection rate revealed a main effect of $\operatorname{TIME}(F(4,132)=15.4, p<$ $\left..001, \eta_{\mathrm{p}}{ }^{2}=.32\right)$, a main effect of $\operatorname{TDCS}\left(F(2,33)=3.2, p=.054, \eta_{\mathrm{p}}{ }^{2}=.16\right)$, and an interaction between TIME and TDCS $\left(F(4,132)=2.15, p=.035, \eta_{\mathrm{p}}{ }^{2}=.12\right)$. The tDCS groups did not differ before and after treatment (all $p$-values $>.1$ ). During treatment, the $\mathrm{L}_{\mathrm{ANODE}}-\mathrm{R}_{\mathrm{CATHOde}}$ group performed better than the sham group, in all trial types (Figure 3), on day $1(t(22)=2.59, p=$ $.042, d=0.998)$, on day $2(t(22)=2.49, p=.053$ and $d=1.016)$ and on day $3(t(22)=3.07, p$ $=.013, d=1.226)$. The $\mathrm{L}_{\mathrm{CATHODE}}-\mathrm{R}_{\mathrm{ANODE}}$ group showed slight improvement compared to the sham group but only on day $3(t(22)=2.36, p=.049, d=0.967)$. The main effect of TRIAL TYPE $\left(F(3,99)=131.73, p<.001, \eta_{\mathrm{p}}^{2}=.80\right)$, and the interaction between TRIAL TYPE and TDCS $\left(F(6,99)=2.238, p=.046, \eta_{\mathrm{p}}{ }^{2}=.12\right)$, indicated that participants committed more errors in bilateral than in unilateral trials and the difference was more pronounced in the sham group. The three-way interaction between TRIAL TYPE, TDCS and TIME was not significant $(F<1)$.

These results support a model including all main effects and an interaction between TIME and TDCS but no three-way interaction with TRIAL TYPE. The Bayesian analysis showed that the data were extremely more likely under this hypothesis than under the null hypothesis $\left(\mathrm{BF}=2.382 * 10^{142}\right)$. The comparison of all models that contain the TIME by TDCs interaction with all models that do not contain the TIME by TDCS interaction yielded a $\mathrm{BF}=1.56$, meaning there was anecdotal evidence for the hypothesis that participants were better at detecting targets 
during the tDCS sessions. The comparison of models with and without the three-way interaction between TIME, TDCS and TRIAL TYPE yielded a BF $=0.013$, meaning there was strong evidence against the hypothesis that target side modulated the tDCS effect. The results of the Bayesian paired t-tests evaluating the group differences per session gave moderate evidence for improved detection in the $\mathrm{L}_{\text {ANODE}}-\mathrm{R}_{\mathrm{CATHODE}}$ group. The comparison of the $\mathrm{L}_{\text {ANODE}}-\mathrm{R}_{\mathrm{CATHODE}}$ group with the sham group yielded BFs $(\mathrm{H} 1 / \mathrm{H} 0)$ of 0.53 pre-tDCS, 2.82 on day $1,3.02$ on day $2,7.06$ on day 3 , and 0.95 after treatment. The comparison of the $\mathrm{L}_{\text {CATHODE- }} \mathrm{R}_{\mathrm{ANODE}}$ group and sham group yielded BFs (H1/H0) of 0.4 pre-tDCS, 0.59 on day $1,0.47$ on day $2,2.52$ on day $3,0.39$ posttDCS. To sum up, left anodal - right cathodal stimulation slightly improved detection, regardless of target side, without producing any after-effect.

\subsection{Response bias}

The analysis of the response bias (left $v s$. right) computed over the number of partial omissions in bilateral trials revealed a main effect of $\operatorname{TDCS}\left(F(2,33)=4.26, p=.023, \eta_{\mathrm{p}}{ }^{2}=.21\right)$. There was no main effect of DAY and no DAY by TDCS interaction $(p>.1)$. Figure 4 suggests that, relative to the baseline performance measured before treatment, participants preferentially reported the target controlateral to the anode when partial omissions occurred in bilateral trials. Between-group comparisons evidenced a modification of the response bias in the $\mathrm{L}_{\mathrm{ANODE}}-$ $\mathrm{R}_{\text {CATHODE }}$ group compared to the sham group (one-tailed $t$-test, $t(11)=2.13, p=.04, d=0.36$ ); the $\mathrm{L}_{\mathrm{CATHODE}}-\mathrm{R}_{\mathrm{ANODE}}$ group did not differ from the sham group (one-tailed $t$-test, $t(11)=0.663$, $p=.256, d=0.11)$. However, a closer look at the data indicated that, in the $\mathrm{L}_{\mathrm{ANODE}}-\mathrm{R}_{\mathrm{CATHODE}}$ group, participants tended to prefer left over right targets before treatment, whereas partial omissions were equally distributed over left and right targets during treatment (see Figure 5). Within-group comparisons showed a modification of the response bias from baseline to tDCS 
sessions in the $\mathrm{L}_{\text {ANODE}}-\mathrm{R}_{\mathrm{CATHODE}}$ group only (one-tailed $t$-test, $t(11)=2.17, p=.026, d=0.627$ ) but this modification cannot be formally interpreted as a normalization of a pre-existing leftward bias, as we found no statistical evidence that the percentage of left ( $v s$. right) responses differed from 0.5 at baseline, neither in the the $\mathrm{L}_{\mathrm{ANODE}}-\mathrm{R}_{\mathrm{CATHODE}}$ group $(t(1)=1.68, p=.121$, $d=0.485)$ nor in the sham group $(t(11)=-1.527, p=.155, d=-0.441)$, nor in the LCATHODE$\mathrm{R}_{\text {ANODE }}$ group $(t(11)=0.693, p=.503, d=0.2)$. Differences in the balance of left-right omissions were still visible post-treatment between $\mathrm{L}_{\mathrm{ANODE}}-\mathrm{R}_{\mathrm{CATHODE}}(-8 \pm 27 \%)$, $\mathrm{L}_{\mathrm{CATHODE}}-$ $\mathrm{R}_{\mathrm{ANODE}}(8 \pm 28 \%)$ and sham treatment $(7 \pm 21 \%)$ but statistical comparisons were not significant (all $p$-values $>.1)$.

The Bayesian analysis of the change in response bias during the tDCS sessions yielded BFs of 49 for the comparison of the $\mathrm{L}_{\mathrm{ANODE}}-\mathrm{R}_{\mathrm{CATHODE}}$ group with the sham group (very strong evidence for a modification of the balance of left-right omissions) and 0.4 for the comparison of the $\mathrm{L}_{\mathrm{CATHODE}}-\mathrm{R}_{\mathrm{ANODE}}$ group with the sham group (anecdotal evidence for no modification). In the post-tDCS session, the same comparisons revealed substantial to strong evidence for an absence of difference between groups $(\mathrm{BF}=0.25$ and $\mathrm{BF}=0.56)$.

\section{Discussion}

The goal of the present study was to assess the potential of bilateral tDCS for attention modulation, as a foundation for the neurorehabilitation of neglect patients. We applied anodal and cathodal stimulation on mirror positions of the left and right PPC of healthy participants performing a visual detection task. The hemispheric rivalry theory predicted that bilateral tDCS should bias attention toward the hemifield controlateral to the anode and thus ipsilateral to the cathode. The results showed that left anodal - right cathodal stimulation improved visual sensitivity to peripheral targets. The balance of left-right omissions revealed no privileged 
processing of one side of space except in trials where left and right targets appeared concurrently, providing partial support to the hemispheric rivalry hypothesis. Below, we discuss the idea that bilateral tDCS enhances visual attention across the full range of space, which may contribute to protect attention from spatial biases especially when the task depends on the well-balanced interaction between hemispheres.

A competition model was useful to account for the effect of non-invasive brain stimulation on the recovery of motor and speech disorders. In these domains, however, the competition model aimed to explain the physiopathology rather than the normal functioning: after stroke, the non-lesioned hemisphere is assumed to exert inhibitory effects on the lesioned hemisphere, which impedes the recovery of the functions normally ensured by the lesioned hemisphere (Turkeltaub, 2015). In the spatial domain, the hemispheric rivalry hypothesis assumes that both hemispheres collaborate to ensure the distribution of attention across the full range of space and spatial biases result from a modification of their ratio of neural activity (Kinsbourne, 1977). The theory provides a valid explanation of the behavioural modification induced by TMS, another neuromodulation technique whose effect on spatial attention has been established by several studies (e.g., Chechlacz et al., 2015; Hilgetag et al., 2001; Koch et al., 2011; Szczepanski and Kastner, 2013). The literature is less clear about the mechanism of action of tDCS. We found that target detection improved on both sides when the anode activated the left hemisphere and the cathode inhibited the right hemisphere. Evidence for directional biases is actually scarce in the literature (Fan et al., 2018; Salazar et al., 2018; Sparing et al., 2009) and the need to move beyond the notion of biased attention was underlined in another research showing that bilateral tDCS did not produce lateralized attention biases but increased the attentional benefits given by a spatial cue presented in advance to the target, irrespective to its side (Duecker et al., 2017). 
We conclude from our results that tDCS has the potential to improve visuospatial processing in neglect patients but that the driving force of such intervention lies in the general enhancement of attention control capacities. The dorsal attention network, formed by the superior parietal and frontal cortex, is known to facilitate the detection of targets for which specific features (e.g., location, the colour or the motion) are known in advance (Corbetta and Shulman, 2002). In our experiment, we assume that tDCS contributed to this function by increasing visual sensitivity to peripheral targets whose eccentricity was constant across trials. Although a subset of parietal (i.e., the ventral intraparietal sulcus) and frontal areas (i.e., the frontal eye fields) selectively respond to the contralateral visual field, paying attention to a cued location generally activates the network bilaterally irrespective to the visual field (Corbetta et al., 2000). Because tDCS was applied in a bilateral mode, we cannot separate the contribution of the right PPC from the contribution of the left PPC in our experiment but previous studies suggest that cathodal stimulation of the right PPC is sufficient to enhance visual attention in a non-lateralized fashion (Moos et al., 2012; Weiss and Lavidor, 2012). Cathodal stimulation is generally associated with impaired performance because of its supposedly predominant inhibitory effect but it has been proposed that, in visuo-spatial tasks, this inhibitory effect might facilitate visual processing by decreasing the global activity of parietal neurons, thus making signal-related activity more distinct (Weiss and Lavidor, 2012). At the neuronal level, there is evidence that the right PPC contains saliency maps coding the position of attended stimuli (Bisley and Goldberg, 2003), and has a remote influence on neural activity in the primary visual cortex (Ruff et al., 2008). The recovery of neglect, from the acute to the chronic stroke stage, has been associated with signal changes in the right PPC during target detection, independently of the visual field, which underlines the interest of using right cathodal stimulation to improve the competence of the right PPC in attention control (Corbetta et al., 2005). 
The asymmetric pattern of left-right omissions in bilateral trials however requires further discussion. We found that left anodal - right cathodal stimulation modified the balance of partial omissions relative to the baseline performance measured before treatment. Despite our attempt to minimize individual spatial biases by calibrating stimulus size, the participants from this group tended to favour left over right targets at baseline. It is possible that spatial biases were not fully controlled in bilateral trials because the calibration was carried out against the detection rate in unilateral - not bilateral - trials. The percentage of partial omissions in bilateral trials could thus be a residual of the strong preference for left vs. right targets that was evident in the calibration (i.e., the titration procedure resulted in smaller sizes for left than right targets). This leftward preference is reminiscent of pseudoneglect, a mild asymmetry in spatial attention that typically favours the left side in young healthy people due to right hemispheric dominance (Benwell et al., 2014; Bowers and Heilman, 1980; Cicek et al., 2009; Jewell and McCourt, 2000; Le et al., 2015; Schmitz and Peigneux, 2011). A reduction of pseudoneglect following left anodal - right cathodal tDCS fits well with the hemispheric rivalry model and with independent evidence from TMS studies combining left PPC excitation with right PPC inhibition (Hilgetag et al., 2001; Petitet et al., 2015). However, it is premature to conclude that bilateral tDCS has the ability to cancel out pre-existing spatial biases because the present study was not designed to assess pseudoneglect and its effect was presumably tuned down by the calibration of stimulus size. Testing this prediction would require tracking the participants' spatial biases throughout treatment, without equalizing performance for left and right targets prior to the experiment. Finally, no leftward deviation was found after left cathodal - right anodal stimulation, possibly reflecting an interaction between electrode montage and functional lateralization of the visuospatial attention network: tDCS would be more effective in shifting attention opposite than along the side of its natural imbalance. Although left cathodal - right anodal tDCS did not affect the imbalance of attention in healthy participants, we expect this 
electrode montage to be effective in neglect patients because they typically display the reversed imbalance at baseline, i.e., a rightward deviation due to hyperexcitability of the left hemisphere after a right lesion. In general, the effect of bilateral PPC-tDCS on lateralized attention biases should be more pronounced in neglect patients than in healthy controls because there is much room for improvement.

To sum up, our results show that bilateral PPC-tDCS has the ability to enhance attention in both visual fields, which may potentially reduce spatial biases in conditions where concurrent stimuli appear at the same time. Clinical studies generally overlook the effects of PPC-tDCS on non-lateralized attention and focus on the search of directional biases, typically in line bisection tasks, which might contribute to underestimate the benefits for neglect rehabilitation (Salazar et al., 2018). The importance of non-lateralized attention deficits in the neglect syndrome is now widely recognized (Corbetta and Shulman, 2011; Husain and Rorden, 2003) and several studies showed that the severity of neglect strongly depends on the amount of attention resources available for spatial monitoring (Cherney and Halper, 2001; Deouell et al., 2005; List et al., 2008; Mennemeier et al., 2004; Ricci et al., 2016; Robertson and Frasca, 1992). Increasing test difficulty or restricting attention resources by adding a non-lateralized dual task dramatically impairs the ability of patients to compensate for their spatial deficit (Bonato et al., 2010; Bonato, 2012). The dual-task approach, in particular, proved successful in revealing neglect or extinction signs, even after three years of chronic stroke, in patients who performed above the cut-off scores on classic tests (Andres et al., 2019; Bonato, 2015). Our study indicates that bilateral tDCS can be used not just to modulate the inter-hermispheric rivalry but also to boost attention resources and create an opportunity window for the learning of compensatory strategies. As in other studies (Giglia et al., 2011; Sparing et al., 2009), we found however that the effects of tDCS were transient. The increased sensitivity to peripheral targets had vanished twenty-four hours after the last tDCS session and the corrective effect on the leftward bias in 
bilateral trials was not significant anymore, even though the stimulation was repeated over three days in sessions of 20 minutes. Moreover, a Bayesian approach to the interaction between time and stimulation indicated that our study only provided moderate evidence for the hypothesis that bilateral tDCS modified the visuospatial behaviour during treatment and no evidence for this hypothesis post-treatment. The translation to clinical trials thus also implies considering other protocols, such as transcranial random noise stimulation (Antal and Herrmann, 2016), that take advantage of the plastic changes induced by stimulation to facilitate functional rehabilitation and maintain the positive outcomes over time. Existing data converge to show that tDCS is more likely to work as an adjuvant rather than as an alternative to standard neglect rehabilitation methods (e.g., Andres and Vandermeeren, 2016; Lefebvre et al., 2013; O'Shea et al., 2017; Panico et al., 2017). 


\section{Conflict of interest statement}

None of the authors have potential conflicts of interest to be disclosed.

\section{Acknowledgements}

MA is a research associate at the Fonds National de la Recherche Scientifique (FRS-FNRS, Belgium). The research was supported by grants T.0245.16 and T.0047.18 from the FRSFNRS. The work of YV was supported by the following grants: FRS-FNRS 1.R.506.16, Fonds de la Recherche Scientifique Médicale (FRSM) 3.4.525.08.F, Fonds Spécial de Recherche (FSR) from the Université catholique de Louvain, Fondation Van Goethem-Brichant, and Fondation Mont-Godinne. 


\section{Figure legends.}

Figure 1. The upper part (A) illustrates the electrode montage in the three groups of participants. According to the hemispheric rivalry hypothesis, participants in the $\mathrm{L}_{\mathrm{ANODE}}-$ $\mathrm{R}_{\mathrm{CATHODE}}$ group should prioritize right target processing whereas participants in the $\mathrm{L}_{\mathrm{CATHODE}}-$ $\mathrm{R}_{\mathrm{ANODE}}$ group should prioritize left target processing. The lower part (B) represents the results of the calibration procedure for one sample participant. The target size was calibrated individually and separately for left (squares) and right (circles) targets after excluding bilateral trials (dotted line). The selected sizes corresponded to adjacent pixel sizes (e.g., $3 \times 3$ and $3 \times 4$ ) giving a detection rate respectively lower and higher than $50 \%$ in calibration trials, thus ensuring that the task engaged a high level of attention resources.

Figure 2. Visual sensitivity. All groups showed increased visual sensitivity during tDCS sessions (from day 1 to 3 - in grey) but this increase was more pronounced in the $\mathrm{L}_{\mathrm{ANODE}}-$ $\mathrm{R}_{\text {CATHODE }}$ group $(\bullet)$ than in the sham $(\square)$ and $\mathrm{L}_{\text {CATHODE}}-\mathrm{R}_{\text {ANODE }}$ groups $(\Delta)$. Error bars represent $95 \%$ confidence intervals.

Figure 3. Correct response rate for the different types of trials before (pre-tDCS), during (from day 1 to 3 - in grey) and after treatment (post-tDCS). The $\mathrm{L}_{\mathrm{ANODE}}-\mathrm{R}_{\mathrm{CATHODE}}$ group is represented by filled circles $(\bullet)$, the $\mathrm{L}_{\mathrm{CATHODE}}-\mathrm{R}_{\mathrm{ANODE}}$ group is represented by open triangles $(\Delta)$, and the sham group by open rectangles $(\square)$. Error bars represent $95 \%$ confidence intervals.

Figure 4. Left-right extinction. The response bias was estimated from partial omissions in bilateral trials and expressed as a difference between tDCS (day 1-3) and baseline (pre-tDCS). A positive value indicates a relative increase in the left (vs. right) response rate compared to baseline, and a negative value indicates a relative increase in the right (vs. left) response rate 
compared to baseline. Participants preferentially reported the target controlateral to the anode when partial omissions occurred in bilateral trials. Error bars represent $95 \%$ confidence intervals.

Figure 5. Bilateral detection before, during and after treatment. LANODE-R $_{\text {CATHODE }}$ stimulation improved the number of correct responses and the balance of left-right omissions in trials where targets appeared on both sides. The percentage of correct bilateral detection is indexed by arrow length. The percentage of partial detection, i.e., 'left only' $v s$. 'right only', is reflected by the leftward vs. rightward vector orientation. A vector aligned with the vertical axis denotes an equal number of left and right omissions among partial responses. The straight lines depict the average performance over the three days of treatment (i.e., with tDCS). The dotted line depicts the performance pre-treatment and the dashed line depicts the performance post-treatment. 


\section{References}

Andres, M., Geers, L., Marnette, S., Coyette, F., Bonato, M., Priftis, K., \& Masson, N. (2019). Increased cognitive load reveals unilateral neglect and altitudinal extinction in chronic stroke. J Int Neuropsychol Soc, 25(6), 644-653.

Andres, M., \& Vandermeeren, Y. (2016). La stimulation cérébrale non-invasive en revalidation. In Traité de Neuropsychologie (Tome 2), X. Seron \& M. Vanderlinden (Eds), Solal: Marseille. Antal, A., \& Herrmann, C. S. (2016). Transcranial alternating current and random noise stimulation: possible mechanisms. Neural plast, 2016, 3616807.

Bisley, J. W., Goldberg, M. E. (2003). Neuronal activity in the lateral intraparietal area and spatial attention. Science, 299, 81-86.

Bonato, M. (2012). Neglect and extinction depend greatly on task demands: A review. Front Hum Neurosci, 6, 195.

Bonato, M. (2015). Unveiling residual, spontaneous recovery from subtle hemispatial neglect three years after stroke. Front Hum Neurosci, 9.

Bonato, M., Priftis, K., Marenzi, R., Umiltà, C., Zorzi, M. (2010). Increased attentional demands impair contralesional space awareness following stroke. Neuropsychologia, 48(13), $3934-3940$.

Bang, D. H., Bong, S. Y. (2015). Effect of combination of transcranial direct current stimulation and feedback training on visuospatial neglect in patients with subacute stroke: a pilot randomized controlled trial. J Phys Ter Sci, 27(9), 2759-2761.

Benwell, C. S., Thut, G., Grant, A., Harvey, M. (2014). A rightward shift in the visuospatial attention vector with healthy aging. Front Aging Neurosci, 6, 113. 
Bowers, D., Heilman, K. M. (1980). Pseudoneglect: Effects of hemispace on a tactile line bisection task. Neuropsychologia, 18(4-5), 491-498.

Brem, A.-K., Unterburger, E., Speight, I., Jäncke, L. (2014). Treatment of visuospatial neglect with biparietal tDCS and cognitive training: A single-case study. Front Syst Neurosci, 8.

Chechlacz, M., Humphreys, G. W., Sotiropoulos, S. N., Kennard, C., Cazzoli, D. (2015). Structural organization of the corpus callosum predicts attentional shifts after continuous theta burst stimulation. J Neurosci, 35(46), 15353-15368.

Cherney, L. R., Halper, A. S. (2001). Unilateral visual neglect in right-hemisphere stroke: A longitudinal study. Brain Injury, 15(7), 585-592.

Çiçek, M., Deouell, L. Y., Knight, R. T. (2009). Brain activity during landmark and line bisection tasks. Front Hum Neurosci, 3, 7.

Corbetta, M., Kincade, M. J., Lewis, C., Snyder, A. Z., Sapir, A. (2005). Neural basis and recovery of spatial attention deficits in spatial neglect. Nat Neurosci, 8(11), 1603.

Corbetta, M., Kincade, J. M., Ollinger, J. M., McAvoy, M. P., Shulman, G. L. (2000). Voluntary orienting is dissociated from target detection in human posterior parietal cortex. Nat Neurosci, 3(3), 292.

Corbetta, M., Shulman, G. L. (2002). Control of goal-directed and stimulus-driven attention in the brain. Nat Rev Neurosci, 3(3), 215-229.

Corbetta, M., Shulman, G. L. (2011). Spatial neglect and attention networks. Annu Rev Neurosci, 34, 569-599.

Dambeck, N., Sparing, R., Meister, I. G., Wienemann, M., Weidemann, J., Töpper, R., Boroojerdi, B. (2006). Interhemispheric imbalance during visuospatial attention investigated by unilateral and bilateral TMS over human parietal cortices. Brain Res, 1072(1), 194-199. 
Denes, G., Semenza, C., Stoppa, E., Lis, A. (1982). Unilateral spatial neglect and recovery from hemiplegia: A follow-up study. Cortex, 105, 543-552.

Deouell, L. Y., Sacher, Y., Soroker, N. (2005). Assessment of spatial attention after brain damage with a dynamic reaction time test. J Int Neuropsychol Soc, 11(6), 697-707.

Di Monaco, M., Schintu, S., Dotta, M., Barba, S., Tappero, R., Gindri, P. (2011). Severity of unilateral spatial neglect is an independent predictor of functional outcome after acute inpatient rehabilitation in individuals with right hemispheric stroke. Arch Phys Med Rehabil, 92(8), $1250-1256$.

Duecker, F., Schuhmann, T., Bien, N., Jacobs, C., Sack, A. T. (2017). Moving beyond attentional biases: Shifting the interhemispheric balance between left and right posterior parietal cortex modulates attentional control processes. J Cogn Neurosci, 29(7), 1267-1278.

Fan, J., Li, Y., Yang, Y., Qu, Y., Li, S. (2018). Efficacy of noninvasive brain stimulation on unilateral neglect after stroke: a systematic review and meta-analysis. Am J Phys Med Rehabil, 97(4), 261-269.

Farnè, A., Buxbaum, L. J., Ferraro, M., Frassinetti, F., Whyte, J., Veramonti, T., et al. (2004). Patterns of spontaneous recovery of neglect and associated disorders in acute right braindamaged patients. J Neurol Neurosur PS, 75(10), 1401-1410.

Giglia, G., Mattaliano, P., Puma, A., Rizzo, S., Fierro, B., Brighina, F. (2011). Neglect-like effects induced by tDCS modulation of posterior parietal cortices in healthy subjects. Brain Stimul, 4(4), 294-299.

Heilman, K. M., Watson, R. T., Valenstein, E. (1993). Neglect and related disorders. In K. M. Heilman E. Valenstein (Eds.), Clinical Neuropsychology (pp. 279-336). Oxford University Press. 
Hilgetag, C. C., Théoret, H., Pascual-Leone, A. (2001). Enhanced visual spatial attention ipsilateral to rTMS-induced virtual lesions of human parietal cortex. Nat Neurosci, 4(9), 953957.

Husain, M., Rorden, C. (2003). Non-spatially lateralized mechanisms in hemispatial neglect. Nat Rev Neurosci, 4(1), 26.

JASP Team. (2018). JASP (Version 0.9) [Computer software]. Retrieved from https://jaspstats.org/

Jeffreys, H. (1961). Theory of probability, (3rd ed.) Oxford, UK: Oxford University Press.

Jewell, G., McCourt, M. E. (2000). Pseudoneglect: a review and meta-analysis of performance factors in line bisection tasks. Neuropsychologia, 38(1), 93-110.

Kinsbourne, M. (1977). Hemi-neglect and hemisphere rivalry. In E. A. Weinstein R. P. Friedland (Eds.), Hemi-inattention and hemisphere specialization (Advances in neurology, pp. 41-49). New York: Raven.

Ko, M.H., Han, S.H., Park, S.H., Seo, J.H., Kim, Y.H. (2008). Improvement of visual scanning after DC brain polarization of parietal cortex in stroke patients with spatial neglect. Neurosci Lett, 448(2), 171-174.

Koch, G., Bonni, S., Giacobbe, V., Bucchi, G., Basile, B., Lupo, F., et al. (2012). Theta-burst stimulation of the left hemisphere accelerates recovery of hemispatial neglect. Neurology, 78(1), 24-30.

Koch, G., Cercignani, M., Bonni, S., Giacobbe, V., Bucchi, G., Versace, V., et al. (2011). Asymmetry of parietal interhemispheric connections in humans. $J$ Neurosci, 31(24), 89678975. 
Koch, G., Oliveri, M., Cheeran, B., Ruge, D., Gerfo, E. L., Salerno, S., et al. (2008). Hyperexcitability of parietal-motor functional connections in the intact left-hemisphere of patients with neglect. Brain, 131(12), 3147-3155.

Koch, G., Veniero, D., Caltagirone, C. (2013). To the other side of the neglected brain: The hyperexcitability of the left intact hemisphere. Neuroscientist, 19(2), 208-217.

Le, A., Stojanoski, B. B., Khan, S., Keough, M., Niemeier, M. (2015). A toggle switch of visual awareness?. Cortex, 64, 169-178.

Lefebvre, S., Laloux, P., Peeters, A., Desfontaines, P., Jamart, J., Vandermeeren, Y. (2013). Dual-tDCS enhances online motor skill learning and long-term retention in chronic stroke patients. Front Hum Neurosci, 6, 1-17.

List, A., Brooks, J. L., Esterman, M., Flevaris, A. V., Landau, A. N., Bowman, G., et al. (2008). Visual hemispatial neglect, re-assessed. J Int Neuropsychol Soc, 14(2), 243-256.

Mennemeier, M. S., Morris, M., Heilman, K. M. (2004). Just thinking about targets can aggravate neglect on cancellation tests. Neurocase, 10(1), 29-38.

Meister, I. G., Wienemann, M., Buelte, D., Grünewald, C., Sparing, R., Dambeck, N., Boroojerdi, B. (2006). Hemiextinction induced by transcranial magnetic stimulation over the right temporo-parietal junction. Neuroscience, 142(1), 119-123.

Moos, K., Vossel, S., Weidner, R., Sparing, R., Fink, G. R. (2012). Modulation of top-down control of visual attention by cathodal tDCS over right IPS. J Neurosci, 32(46), 16360-16368. Oliveri, M., Bisiach, E., Brighina, F., Piazza, A., La Bua, V., Buffa, D., et al. (2001). rTMS of the unaffected hemisphere tran- siently reduces contralesional visuospatial hemineglect. Neurology, 57(7), 1338-40. 
O'Shea, J., Revol, P., Cousijn, H., Near, J., Petitet, P., Jacquin-Courtois, S., et al. (2017). Induced sensorimotor cortex plasticity remediates chronic treatment-resistant visual neglect. Elife, 6, e26602, 1-38.

Panico, F., Jacquin-Courtois, S., Di Marco, J., Perrin, C., Trojano, L., Rossetti, Y. (2017). tDCS reactivation of dormant adaptation circuits. Cortex, 94, 196-199.

Peirce, J. W. (2007). PsychoPy_Psychophysics software in Python. J Neurosci Methods, 162, $8-13$.

Petitet, P., Noonan, M. P., Bridge, H., O’Reilly, J. X., O’Shea, J. (2015). Testing the interhemispheric competition account of visual extinction with combined TMS/fMRI. Neuropsychologia, 74, 63-73.

Ricci, R., Salatino, A., Garbarini, F., Ronga, I., Genero, R., Berti, A., Neppi-Mòdona, M. (2016). Effects of attentional and cognitive variables on unilateral spatial neglect. Neuropsychologia, 92, 158-166.

Robertson, I.H, Frasca, R., 1992. Attentional load and visual neglect. Int J Neurosci, 62, 4556.

Rode, G., Fourtassi, M., Pagliari, C., Pisella, L., Rossetti, Y. (2017). Complexity vs. unity in unilateral spatial neglect. Rev Neurol (Paris), 1-11.

Ruff, C. C., Bestmann, S., Blankenburg, F., Bjoertomt, O., Josephs, O., Weiskopf, N., et al. (2008). Distinct causal influences of parietal versus frontal areas on human visual cortex: Evidence from concurrent TMS-fMRI. Cereb Cortex, 18(4), 817-827.

Salazar, A. P. S., Vaz, P. G., Marchese, R. R., Breitenstein, C., Pinto, C., Pagnussat, A. S. (2018). Noninvasive brain stimulation improves hemispatial neglect after stroke: A systematic review and meta-analysis. Arch Phys Med Rehabil, 99(2), 355-366. 
Schmitz, R., Peigneux, P. (2011). Age-related changes in visual pseudoneglect. Brain Cogn, $76(3), 382-389$.

Smit, M., Schutter, D. J., Nijboer, T. C., Visser-Meily, J. M., Kappelle, L. J., Kant, N., et al. (2015). Transcranial direct current stimulation to the parietal cortex in hemispatial neglect: a feasibility study. Neuropsychologia, 74, 152-161.

Song, W., Du, B., Xu, Q., Hu, J., Wang, M., Luo, Y. (2009). Low-frequency transcranial magnetic stimulation for visual spatial neglect: A pilot study. J Rehabil Med, 41(3),162-5.

Sparing, R., Thimm, M., Hesse, M. D., Kust, J., Karbe, H., Fink, G. R. (2009). Bidirectional alterations of interhemispheric parietal balance by non-invasive cortical stimulation. Brain, 132(11), 3011-3020.

Sunwoo, H., Kim, Y. H., Chang, W. H., Noh, S., Kim, E. J., Ko, M. H. (2013). Effects of dual transcranial direct current stimulation on post-stroke unilateral visuospatial neglect. Neurosci Let, 554, 94-98.

Szczepanski, S. M., Kastner, S. (2013). Shifting attentional priorities: control of spatial attention through hemispheric competition. J Neurosci, 33(12), 5411-5421.

Turgut, N., Miranda, M., Kastrup, A., Eling, P., Hildebrandt, H. (2018). tDCS combined with optokinetic drift reduces egocentric neglect in severely impaired post-acute patients. Neuropsychol Rehab, 28(4), 515-526.

Turkeltaub, P. E. (2015). Brain stimulation and the role of the right hemisphere in aphasia recovery. Curr Neurol Neurosci Rep, 15(11), 72.

Vossel, S., Weiss, P. H., Eschenbeck, P., Fink, G. R. (2013). Anosognosia, neglect, extinction and lesion site predict impairment of daily living after right-hemispheric stroke. Cortex, 49(7), $1782-1789$. 
Wagenmakers, E. J., Marsman, M., Jamil, T., Ly, A., Verhagen, J., Love, J., et al. (2018). Bayesian inference for psychology. Part I: Theoretical advantages and practical ramifications. Psychon Bull Rev, 25(1), 35-57.

Weiss, M., Lavidor, M. (2012). When less is more: Evidence for a facilitative cathodal tDCS effect in attentional abilities. J Cogn Neurosci, 24(9), 1826-1833. 


\section{Figure 1.}

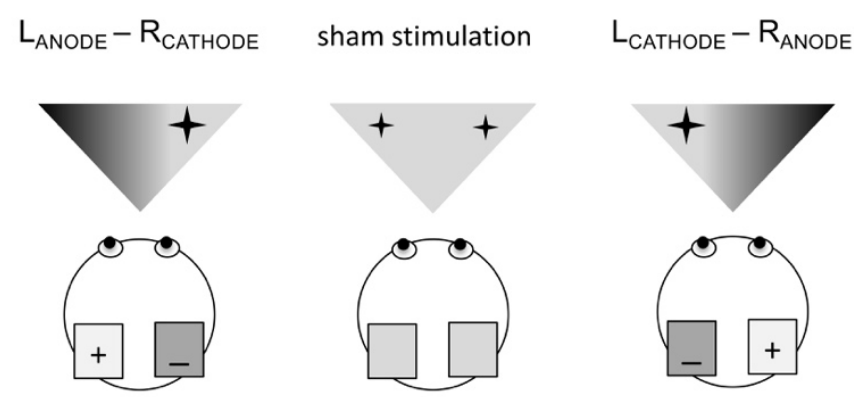

B Correct response rate in calibration trials

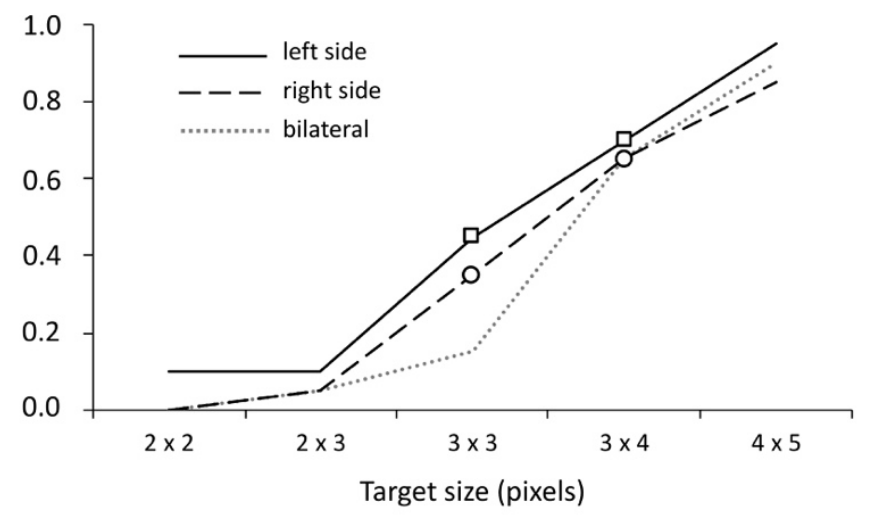


Figure 2.

Visual sensitivity index ( $\left.d^{\prime}\right)$

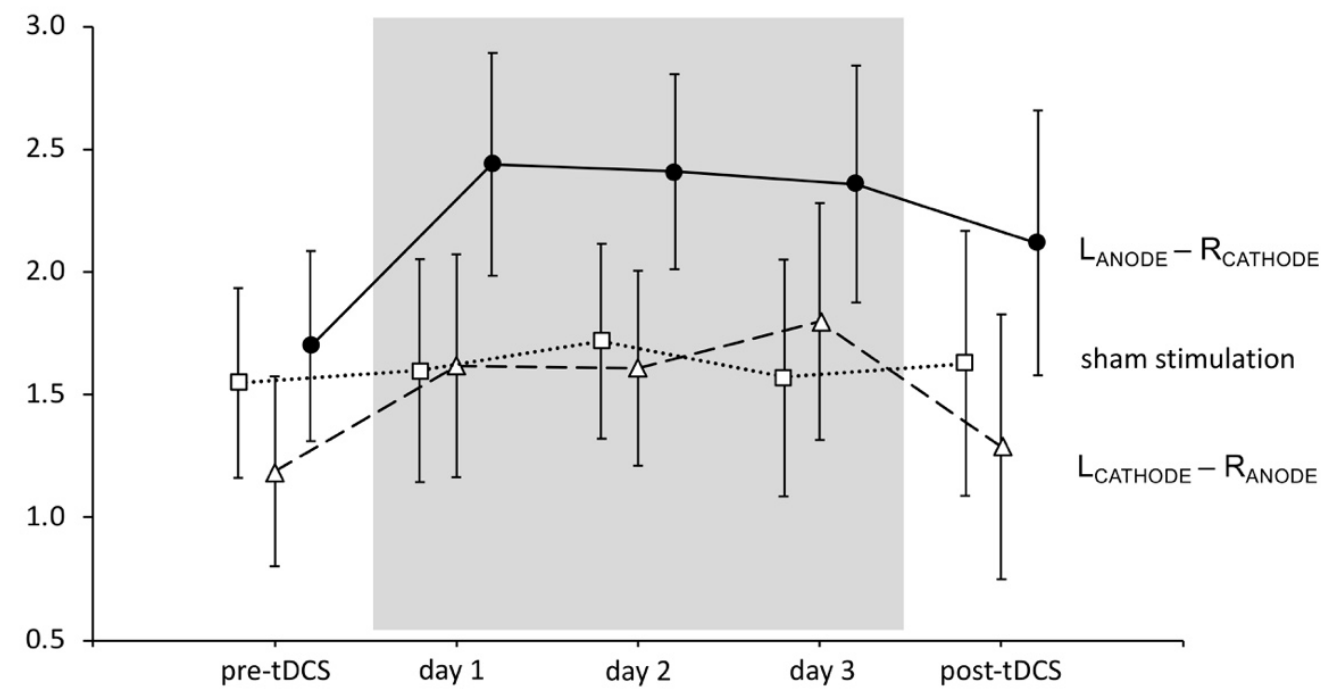




\section{Figure 3.}
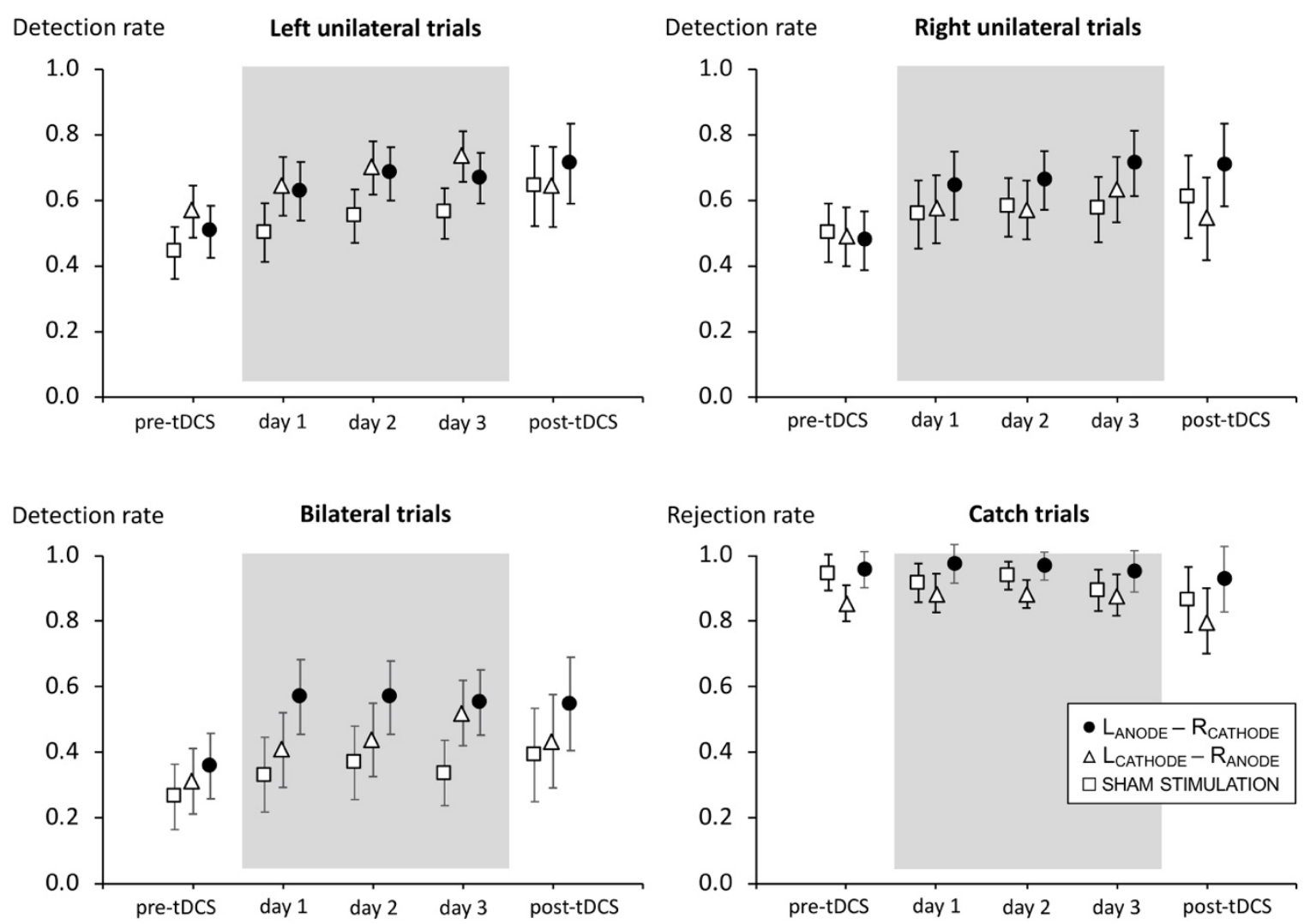
Figure 4.

Difference in \% of left responses

relative to baseline
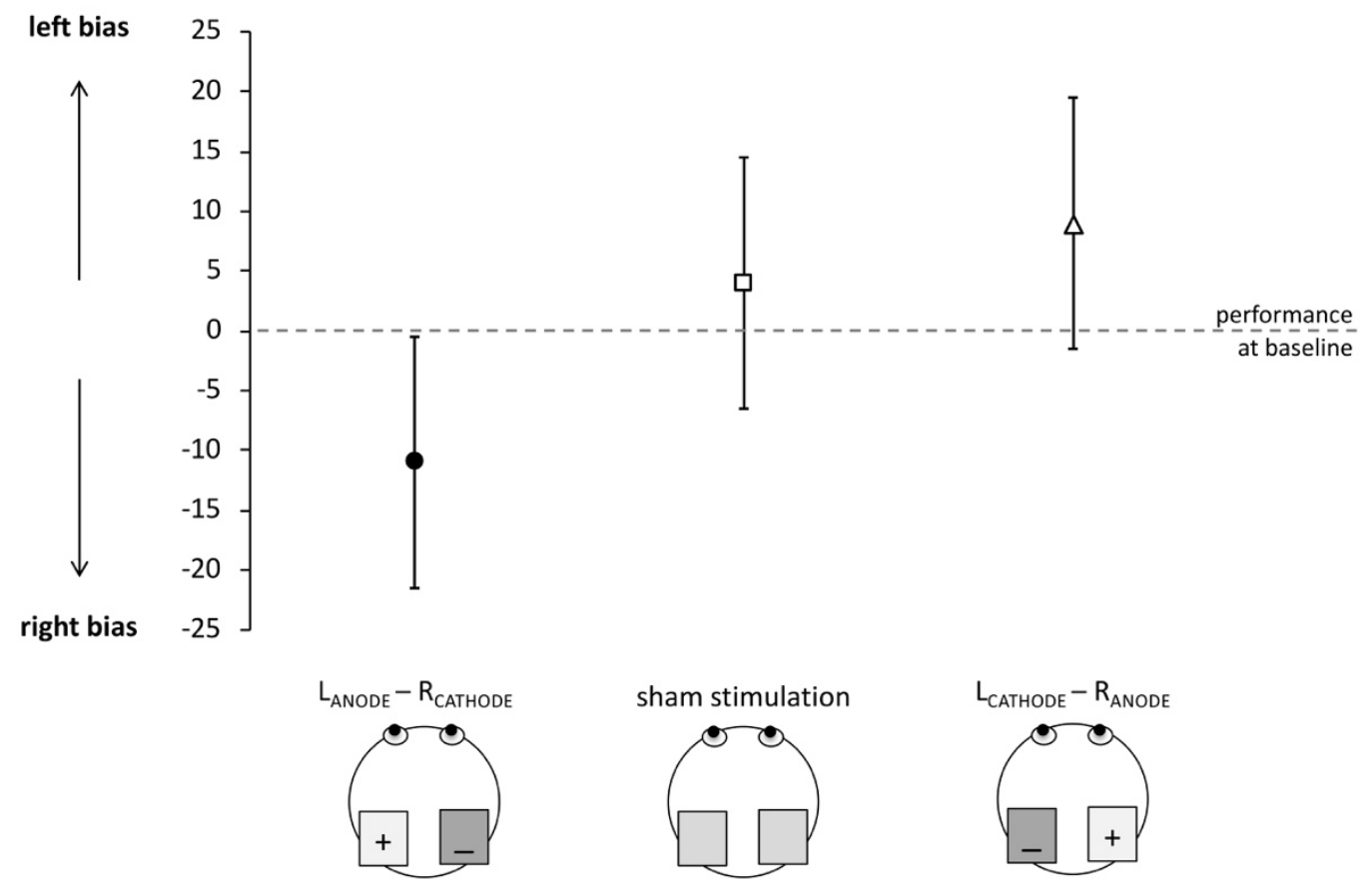


\section{Figure 5.}
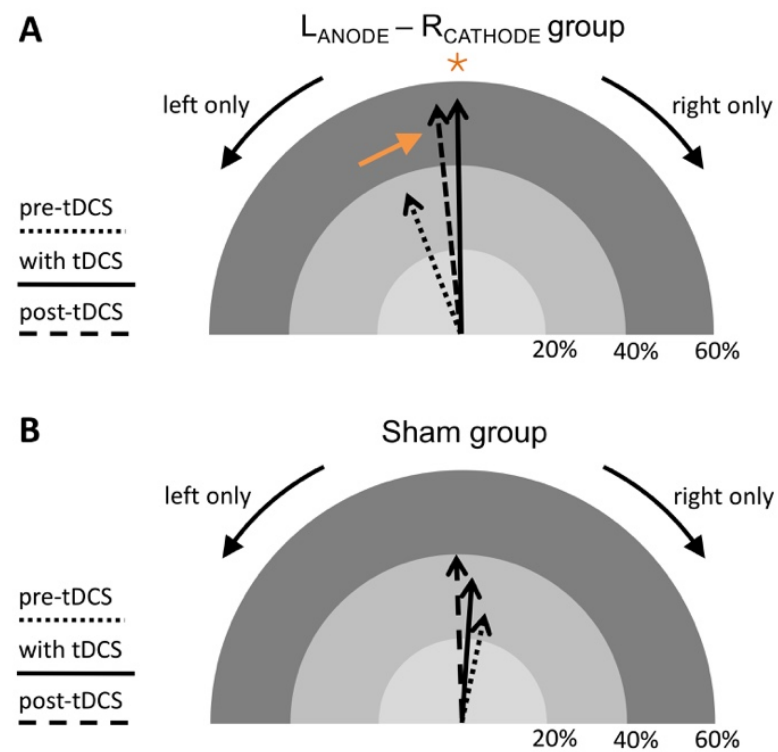

C $\mathrm{L}_{\text {CATHODE }}-\mathrm{R}_{\mathrm{ANODE}}$ group

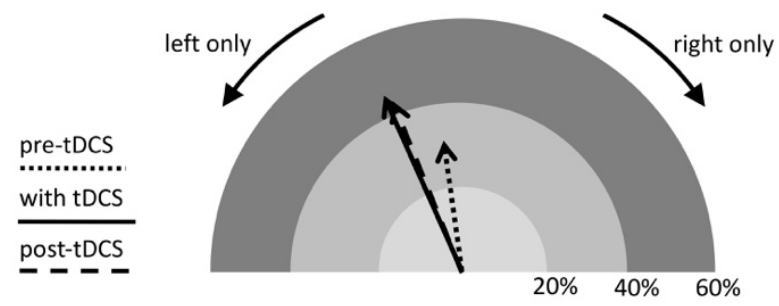

